\title{
Fundamental Investigations in the Design of Five-Axis Nanopositioning Machines for Measurement and Fabrication Purposes
}

\author{
Ralf Schienbein $^{1}$ (i) $\cdot$ Florian Fern ${ }^{2} \cdot$ René Theska $^{1}$ (i) $\cdot$ Shraddha Supreeti $^{3} \cdot$ Roland FüßI $^{2} \cdot$ Eberhard Manske $^{2}$ (i)
}

Received: 21 December 2020 / Revised: 15 February 2021 / Accepted: 25 February 2021 / Published online: 20 April 2021

(C) The Author(s) 2021, corrected publication 2022

\begin{abstract}
The majority of nanopositioning and nanomeasuring machines (NPMMs) are based on three independent linear movements in a Cartesian coordinate system. This in combination with the specific nature of sensors and tools limits the addressable part geometries. An enhancement of an NPMM is introduced by the implementation of rotational movements while keeping the precision in the nanometer range. For this purpose, a parameter-based dynamic evaluation system with quantifiable technological parameters has been set up and employed to identify and assess general solution concepts and adequate substructures. Evaluations taken show high potential for three linear movements of the object in combination with two angular movements of the tool. The influence of the additional rotation systems on the existing structure of NPMMs has been investigated further on. Test series on the repeatability of an NPMM enhanced by a chosen combination of a rotary stage and a goniometer setup are realized. As a result of these test series, the necessity of in situ position determination of the tool became very clear. The tool position is measured in situ in relation to a hemispherical reference mirror by three Fabry-Pérot interferometers. FEA optimization has been used to enhance the overall system structure with regard to reproducibility and long-term stability. Results have been experimentally investigated by use of a retroreflector as a tool and the various laser interferometers of the NPMM. The knowledge gained has been formed into general rules for the verification and optimization of design solutions for multiaxial nanopositioning machines.
\end{abstract}

Keywords Multiaxial nanopositioning and nanomanufacturing · Ultra-precision machine designs · 5-Axis operation · Ultraprecision rotations

Ralf Schienbein

ralf.schienbein@tu-ilmenau.de

René Theska

rene.theska@tu-ilmenau.de

Eberhard Manske

eberhard.manske@tu-ilmenau.de

1 Department of Mechanical Engineering, Institute of Design and Precision Engineering, Technical University of Ilmenau, Ilmenau, Germany

2 Institute of Process Measurement and Sensor Technology, Department of Mechanical Engineering, Technical University of Ilmenau, Ilmenau, Germany

3 Electronics Technology Group, Department of Electrical Engineering and Information Technology, Technical University of Ilmenau, Ilmenau, Germany

\section{Introduction}

As result of a comprehensive literature survey, the majority of coordinate measuring machines with a repeatability in the nanometer range (nanopositioning and nanomeasuring machines, NPMMs) are based on three independent linear movements of up to $400 \mathrm{~mm} \times 400 \mathrm{~mm} \times 100 \mathrm{~mm}(x y z)$ in a Cartesian coordinate system [1-6]. This, in combination with the specific nature of sensors and tools (further on summarized as tool), limits the addressable part geometries. Depending on the tool in use, spherical and aspherical geometries as well as free-form surfaces cannot be measured or only to a certain limit. This article contributes to the enhancement of multiaxial machine structures by the implementation of rotational movements while keeping the precision untouched. Approaches are known that allow the movement with a degree of freedom of four or five, which is required for addressing freeform surfaces orthogonal to the surface [7-9]. These approaches do not achieve nanometer 
precision for working ranges of NPMMs. In comparison to this, the aim of this work is the extension of NPMMs with additional degrees of rotational freedom for multitool concepts and freeform manufacturing in working areas of up to $200 \mathrm{~mm} \times 200 \mathrm{~mm} \times 25 \mathrm{~mm}[10,11]$. Suitable concepts of the overall machine structure were investigated. A parameter-based evaluation system was created to identify solution concepts fulfilling given requirements [12]. The rotation of the tool in use showed a high application potential. This article contributes to the systematic development of multiaxial machine structures based on existing NPMMs $[10,11]$ in the early design state.

\section{Overall Structural Concepts}

\subsection{Evaluation of Multiaxial NPMMs}

To support the selection of machine structures for the development of a multiaxial nanopositioning machine, an evaluation system was developed in accordance with the VDI guideline 2225 [5, 13, 14]. This is based on modular arrangement variants of basic components, a dynamic evaluation system, and indications of the characteristics to be considered for the design process.

The fundamental structures of Cartesian nanopositioning and nanomeasuring machines are based on three modes of movement: the sample scanning mode (SSM), the mixed scanning mode (MSM), and the probe scanning mode (PSM). The movement of the object or the tool is measured on the base of a reference with highest thermal and geometric stability fulfilling the Abbe Comparator Principle.

Suitable concepts of the extension of pure Cartesian structures with additional degrees of rotational freedom were investigated $[5,13]$. In addition, compliance with the Abbe Comparator Principle has been an indispensable prerequisite for the design of the multiaxial NPMM. First evaluations for the overall structure show the highest degree of fulfilment for an SSM with additional rotations (turns and tilts) of the tool (Fig. 1b). Alternatively, rotations of the sample or of the sample and the tool have been considered, as shown in Fig. 1.

The integration of additional rotary positioning systems near the respective end effector (object or tool) within the functional chain between the base and the end effector leads to:

- Reduced masses to be moved and thus increased dynamic and reduced heat input.

- Reduced complexity of the measuring system and the corresponding reference for comparatively large translational $(\geq 10 \mathrm{~mm})$ and rotational $\left(\geq 10^{\circ}\right)$ movements for the fulfilment of the Abbe Comparator Principle.

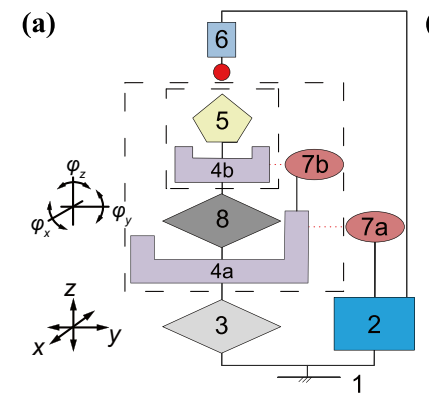

(b)
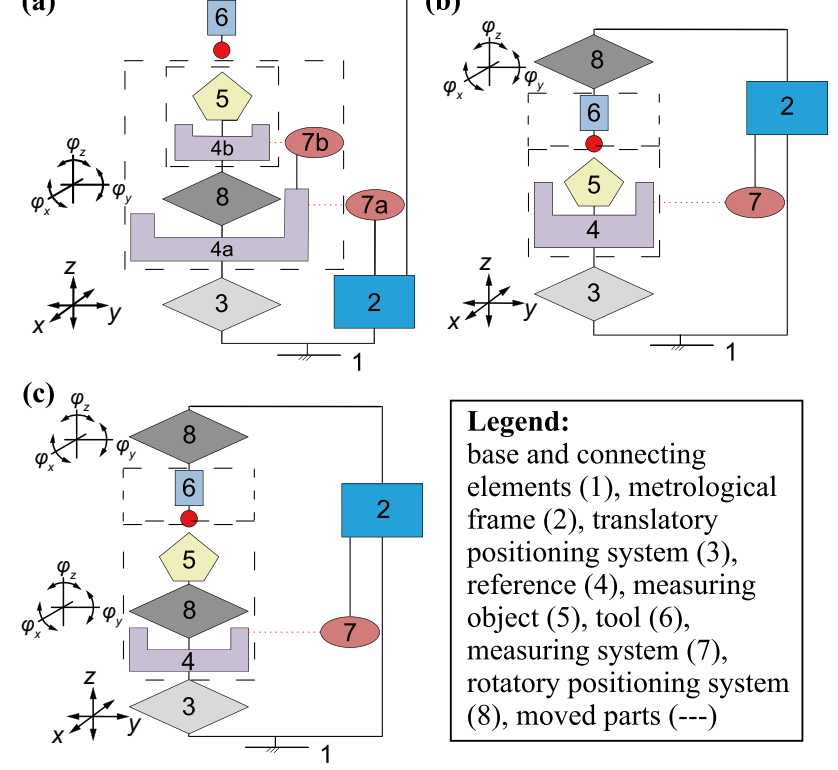

\section{Legend:}

base and connecting

elements (1), metrological

frame (2), translatory

positioning system (3),

reference (4), measuring

object (5), tool (6),

measuring system (7),

rotatory positioning system

$(8)$, moved parts (---)

Fig. 1 System configurations for the sample scanning mode with additional rotations of the object (a), the tool (b), or tool and object (c)

\subsection{Sample Scanning Mode with Additional Tool Rotations}

A central part of the fundamental structure of a positioning system is the underlying kinematic, which has a decisive influence on the design of the drive system.

In a first step, the rotation of a representative tool with a maximum mass of $m_{T} \leq 2 \mathrm{~kg}$, an overall extension in the measuring direction of $l_{T} \leq 60 \mathrm{~mm}$ and rotation angles of $90^{\circ}$ for $\varphi_{x, y}$ and $360^{\circ} \varphi_{z}$ are considered. For this purpose, a total of 21 suitable kinematic variants in order to address a hemisphere orthogonal to its surface are determined by means of a structural synthesis. The position of the instantaneous center of rotation, the number of joints, and the orientation of the rotary axes to the main axes of the NPMM have been varied.

In the following, kinematic variants are selected by use of a parameter-based evaluation system [5]. The evaluation criteria deployed are mainly based on quantitative characteristics. These include the complexity (number of joints), the position of the instantaneous center of rotation, expected masses and angles of rotation as well as the dependence of the axes of rotation to each other. To achieve this by means of commercially available positioning systems for ultra-precise rotations including multiaxial solutions such as hexapods [15], characteristic typical values of positioning systems are derived from comprehensive design catalogues [13]. Software-supported [16] parameter studies are used to derive typical dimensions 


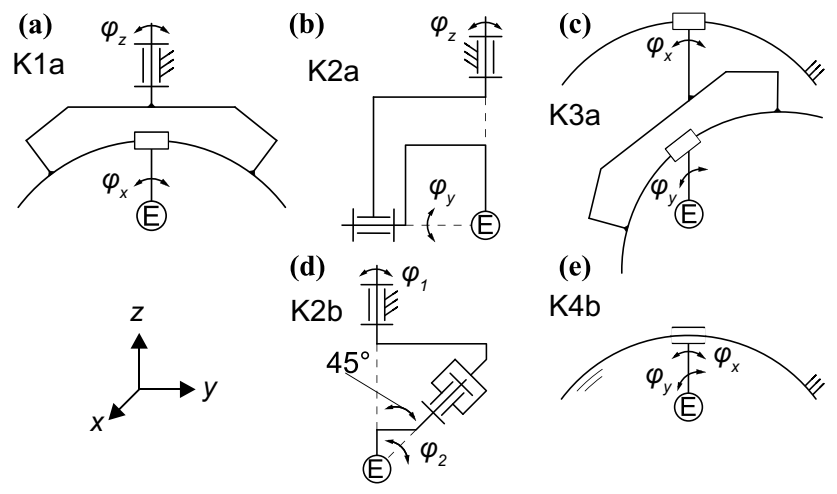

Fig. 2 Suitable kinematics for tool rotations

(a)

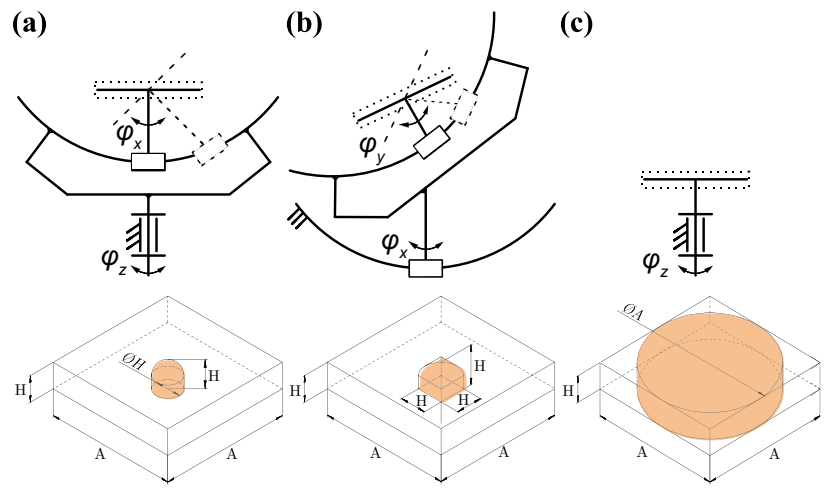

Fig. 3 Restrictions of object geometries due to the concept of object rotation

and motion ranges. Figure 2 shows the kinematics with the highest degree of fulfilment.

These variants $\mathrm{K} 1 \mathrm{a}-\mathrm{K} 4 \mathrm{a}$ describe rotations of the end effector (E) as a tool around the main axes of the Cartesian coordinate system with a common instantaneous center of rotation in the tool center point (TCP).

From the investigation it could be deduced that those kinematics are generally the most suitable. The tool rotation in form of a hemisphere is realized by rotation angles of $90^{\circ}$ for $\varphi_{x, y}$ and $360^{\circ} \varphi_{z}$. Linear corrective movements are not necessary since the tool center point coincides with the Abbe Point, thus avoiding first-order positioning errors.

\subsection{Sample Scanning Mode with Additional Sample Rotations}

In analogy to the rotation of the tool, possible kinematic variants are determined and evaluated for the rotation of the sample for addressing a hemisphere orthogonally to its surface. Since the sample has an unknown structure, linear correction movements are necessary to address the whole sample surface. As a result, the addressable sample points or the angle of rotation of the positioning system used, are considerably restricted. The sample dimensions are limited to a subset of the Cartesian volume. Depending on the kinematic variants, characteristical addressable volumes are showing the limits for given rotation kinematics (Fig. 3). If the sample fits to the dimensions derived from the given Cartesian volume, any hemisphere within the characteristic volume can be addressed.

The added mass and the necessary power supply of the rotary positioning systems must be taken into account for the design of the linear positioning systems. The deformation of the object when changing its orientation in the gravitational field remains as an undetermined influence. An adaptation of the object shape cannot be assumed, which is particularly important for nanofabrication processes.

In comparison with object rotations, the rotary positioning systems and corresponding coupling elements can be adapted to the tool design. This enables optimizations with regard to minimum mechanically and thermally induced deformations or their consideration within a tool-specific calibration process.

The solution variants for the rotation of the tool show in comparison the higher degree of fulfilment.

\subsection{Sample Scanning Mode with Additional Sample and Tool Rotations}

The restrictions of object geometries can be reduced to the level of one individual rotary axis (Fig. 3b), if rotations of the tool are implemented in addition to the object movements.

However, a higher degree of freedom influences the achievable uncertainty of the NPMM [7]. If the exact position of the tool center point (TCP) is not measured directly, the displacement as a consequence of the error motion can be calculated by geometric dimensions of the substructures. There are three translational and three rotational errors for every stage of rotation, which are dependent on the actuation angle. The movement of the TCP can be calculated by a superposition of the components resulting from those error motions. For tool rotation principles with an instantaneous center of rotation in the TCP, the resolution and measurement uncertainty of the rotation measurement system does not affect the position of the TCP.

The sample rotation can be described in the same manner as for the tool rotation. Since the TCP is fixed, the error motion of the positioning system now affects the position of the sample. Due to the dimension of the sample, the sample position is not independent from the properties of the angular measurement system. Although the characteristic lengths are much smaller for the sample rotation, the described systematic error leads to a 
comparable uncertainty for both approaches [17]. One approach to reduce the measurement uncertainty of the overall system is the in situ measurement of the respective translation and rotation errors of the rotation systems. This is simplified by the separation of the rotations to the object and tool, since only one positioning system has to be measured.

\subsection{Investigation of the Positioning Repeatability}

Best position repeatability is an indispensable prerequisite to secure the measurement uncertainty of the NPMM. If that is given, the remaining deviations can be corrected based on calibration data. A combination of a rotary stage [18] and a goniometer [19] for two independent rotations of the tool of an SSM-based NPMM has been chosen as the setup in specific (Fig. 4). This allows a tool $\left(l_{T} \leq 60 \mathrm{~mm} ; m_{T} \leq 2 \mathrm{~kg}\right)$ to be tilted horizontally by up to $90^{\circ}$ and rotated $360^{\circ}$ around the vertical axis of the NPMM.

Measurement series are required to take the positioning repeatability of the rotation into account. The overall positioning repeatability of the tool can be derived by the combination of those of the individual axes or by measuring at the end effector (tool) directly. For this purpose, the positioning deviations of the rotation axes (Fig. 4) were measured with a universal autocollimator [21] along the respective axis with regard to the sensitivity to different stage setups (standing, hanging, single, or combined stages).

No significant changes of the repeatability caused by the arrangement were found. The repeatability depends on the angular position and the design of the positioning system. For the rotary stage in a setup equivalent to Fig. 4, the highest repeatability is $\leq \pm 14.42 \mu \mathrm{rad}$ within the positioning range. For the goniometer, the same conditions result in $\leq \pm 17.95 \mu \mathrm{rad}$. These values are several orders of

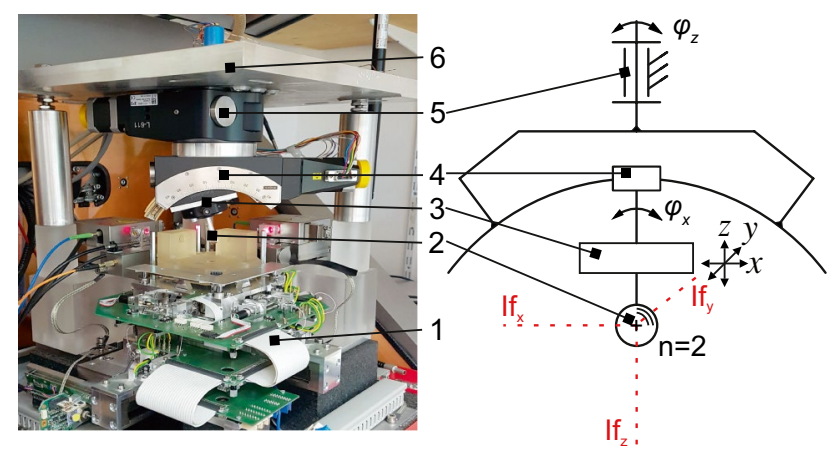

Fig. 4 NPMM [11] inside a climatic chamber with additional tool rotations; 1: linear stage of the $\operatorname{NPMM}(x, y, z), 2$ : glass sphere $(n=2)$ in the TCP, 3: tool adjuster $(x, y, z)[20], 4$ : goniometer stage $\left(\varphi_{x, y}\right)$ [19], 5: rotary stage $\left(\varphi_{z}\right)$ [18], 6: metrological frame, (If) interferometer magnitude lower than the expected sensitivity of tools for surface inclinations of $\geq 1^{\circ}$ to the tool axis, above which a significant increase in their measurement uncertainty can be expected [9].

For the measurement of the combined rotational movement around two axes, a glass sphere with a refractive index of 2 was used as a retroreflector placed in the TCP (Fig. 4) [22]. After removal of the linear stage, the three laser interferometers of the NPMM were used to determine the lateral deviations of the TCP while the tool was rotated over the full range. The goniometer is used to set the angular positions of the ball shaft to the vertical in steps of $\Delta \varphi_{x, y}=5^{\circ}$. In addition, the rotary stage rotates in steps of $\Delta \varphi_{z}=30^{\circ}$ resulting in 91 measuring positions. The individual position is held for $60 \mathrm{~s}$ to reduce the influence of vibration. Long-term measurements of ten repetitions over $10 \mathrm{~h}$ are carried out for the entire series of positioning repeatability measurements inside the closed climate chamber of the NPMM.

The best-measured position repeatability over the entire rotation range and all measurement series is $\pm 100 \mathrm{~nm}$ for the $x$ - and $y$-direction and $\pm 150 \mathrm{~nm}$ in the $z$-direction [5].

\section{Tool Rotation with In Situ Deviation Measurement}

The measurement and machining accuracy of the multiaxial NPMM depends on the positioning accuracy. The achievable accuracy is limited by the repeatability of the positioners. To overcome this, in situ measurement of the tool rotation based on a calibrated reference needs to be implemented. A parameter-based dynamic evaluation system with quantifiable technological parameters has been performed to identify general solution concepts [5, 14]. The number, arrangement, and operating principle of the measuring system and its reference were systematically investigated. Structural variants with a high degree of fulfilment consider the position detection at the coupling point of the end effector (reference) in order to reduce the number of necessary measuring systems and thus the total measurement uncertainty (Fig. 5).

Adapted from the design of Cartesian NPMM, the measuring beams are reflected at the corresponding reference, and intersect virtually at a point that coincides with the TCP thus fulfilling the Abbe Comparator Principle. Out of the evaluations, a system of three laser interferometers attached to the tool in correspondence to a hemispherical concave mirror as reference, attached to the metrological frame of the NPMM (Fig. 5b) has been chosen.

The developed design enables the strict separation of the force frame and the metrological frame. Further on, the thermal shielding of the rotary stage being the largest heat source (Fig. 6) can be realized. The hemisphere stands on 


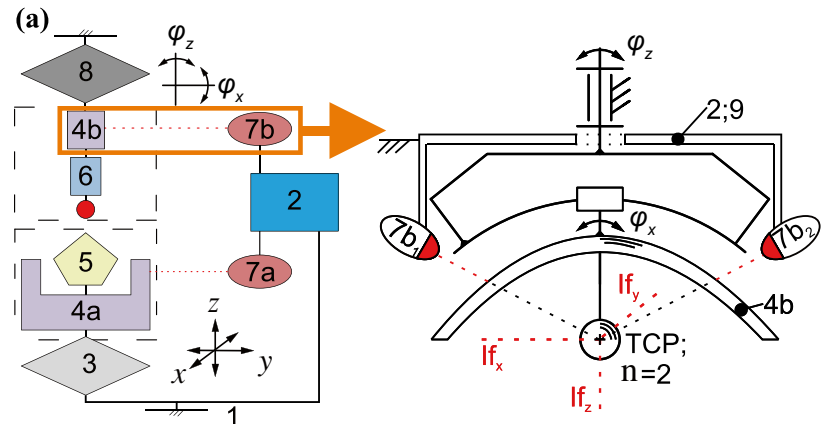

(b)

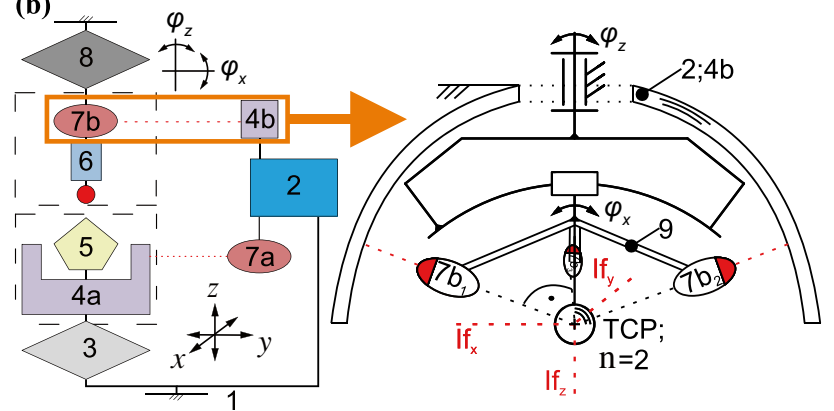

Fig. 5 System configurations for the SSM with additional tool rotations and in situ deviation measurement with rotations of the reference (a), or of the measuring system (b), (components see Figs. 1 and 4 , interferometer carrier IC (9))
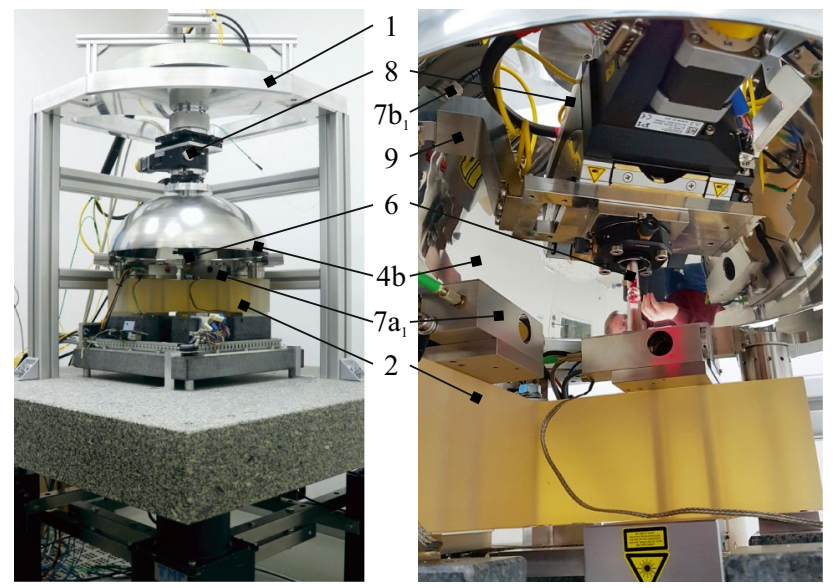

Fig. 6 SSM-based NPMM [11] with additional tool rotations [18, 19] and in situ deviation measurement with rotations of three Fabry-Pérot interferometers [23] and a reference hemisphere as part of the metrological frame of the NPMM, (for components see Fig. 5)

a support made of Invar36 and can be adjusted in the main axes of the NPMM as well as subsequently fixed in position with minimized thermally induced stress. The three Fabry-Pérot interferometers are connected by a metrological frame and form a Cartesian coordinate system with their measuring axes, which are matching virtually in the TCP.
This way, the rotational positioning systems can be included in the metrological loop via an in situ measurement of the displacements of the TCP.

In addition, the positioning systems are connected to the base via a separate force frame, which reduces the mechanically induced deformations in the metrological frame. Alignment and subsequent position assurance of the positioning systems, the Fabry-Pérot interferometers, and the tool are done in each case via elements allowing adjustment along the main axes of the NPMM.

Since the hemisphere is used as a reference, the remaining unavoidable shape deviations must be determined. Due to the large diameter of the reference surface of $400 \mathrm{~mm}$ and the comparatively strong curvature, the shape deviations are determined in situ.

Using a glass sphere with a refractive index of two in the $\mathrm{TCP}$, an in situ calibration is done by means of the three laser interferometers of the NPMM. The measured values of the Fabry-Pérot interferometers are assigned to the measured values of the NPMM laser interferometers simultaneously during the rotation of the tool. A first proof of concept has been realized on the basis of a none perfect but affordable reference hemisphere of polished stainless steel (Fig. 6).

Figure 7 shows an example of the measured values for the interferometers of the NPMM (Fig. 7a) and the corresponding values of the Fabry-Pérot interferometers (Fig. 7b) determined simultaneously during the rotation around the $z$-axis of the NPMM. The values are shown in the direction of the respective measurement axes for repeated forward and backward rotations (fwd/bwd).
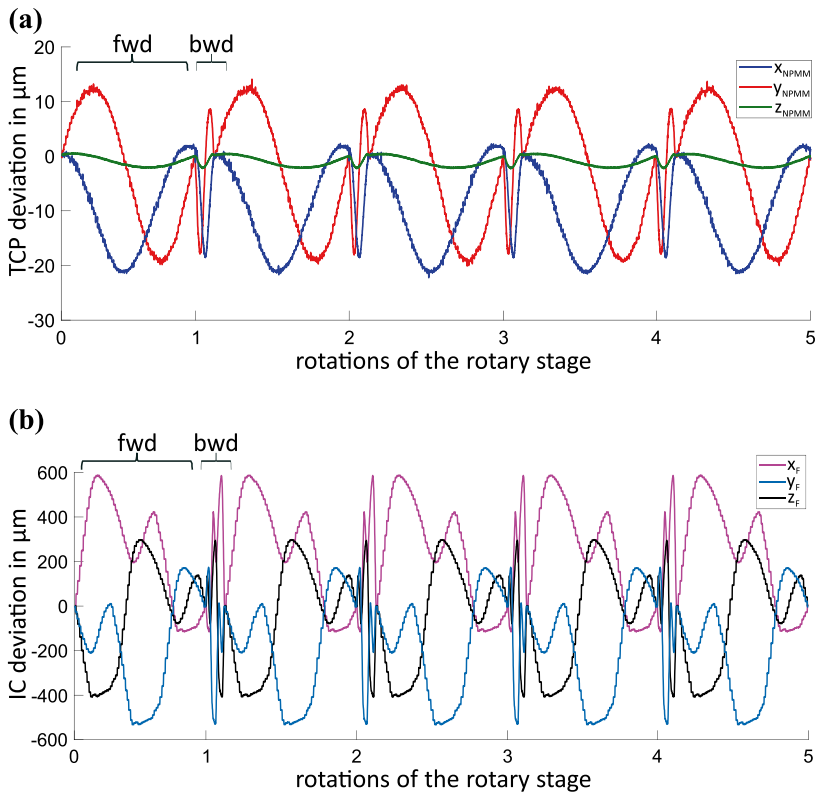

Fig. 7 Measured values of the six interferometers for the relative movements of the TCP with respect to the metrological frame 
The comparatively large measured values of the Fabry-Pérot interferometers are mainly caused by the shape deviations in the range of $\pm 350 \mu \mathrm{m}$ of the stainless-steel hemisphere. For repeated measurements between individual positions of the rotary stage, the TCP could be determined with a deviation down to a maximum of $27 \mathrm{~nm}$, which is already in the range of the periodic nonlinearities of the Fabry-Pérot interferometers $( \pm 13 \mathrm{~nm})$.

Within the framework of a detailed theoretical measurement uncertainty analysis for a setup with a hemisphere made of $\mathrm{SiO}_{2}$ (fused silica), the determination of the TCP could be proven with a maximum measurement uncertainty of $\leq 18 \mathrm{~nm}(p=68 \%)$ in each direction $(x, y, z)$ [24]. The largest contributions to the uncertainty budget are given by the thermal stability of the reference hemisphere and, after the material change to fused silica, by the orthogonality deviations and the nonlinearities of the Fabry-Perot interferometers.

These compact interferometers allow measurements on both highly curved surfaces of high reflectivity, such as polished metals, and transparent surfaces of low reflectivity, such as fused silica [24].

As part of the static metrological frame, the hemisphere is to be designed as a reference for the interferometers with regard to highest long-term stability and thus minimal thermally and mechanically induced deformations (Table 1). The mechanically induced deformation can be reduced in particular by increasing the wall thickness in the area of the contact surface of the standing reference hemisphere (variants 1-2a and 2-2a in Table 1).

Reduced mechanically-induced deformations in turn decrease the creep of these structures, as an additional deformation $\varepsilon(t)$, relative to the elastic start deformation $\varepsilon_{0}$. For titanium silicate glass (ULE) and fused silica, for example, after $t=500 \mathrm{~h}$ values of $\varepsilon(t)$ of $\leq 0.1 \%$, for Invar $36 \leq 0.039 \%$ and for the glass ceramic Zerodur $\leq 0.7 \%$ can be assumed [6].

\section{Nanofabrication with a Rotating Stamp of Nanoimprint Lithography}

As was to be expected, the existing measurement uncertainties of the NPMM are slightly increased by additional rotary positioning systems. However, a scientific novelty is the possibility of addressing and processing three-dimensional bodies normal to their surface including undercuts. The alignment of a tool for nanoimprint lithography (NIL) normal to a strongly curved surface through the developed tool rotation of the NPMM opens up new application possibilities in the field of nanofabrication (Fig. 8).

The integrated NIL tool is developed based on the basic components of soft UV-NIL. It consists of a UV LED light source with a wavelength of $365 \mathrm{~nm}$ and an intensity of $148 \mathrm{~mW} / \mathrm{cm}^{2}$ [25]. A fused silica plano-convex lens is used to focus the light on an area of approximately $20 \mathrm{~mm}^{2}$. The focused light is transmitted through the patterned area of $25 \mathrm{~mm}^{2}$ on the PDMS (polydimethylsiloxane, Sylgard 184 silicone elastomer) stamp. The stamp of 20-mm diameter has been fixed to the tip of the NIL tool.

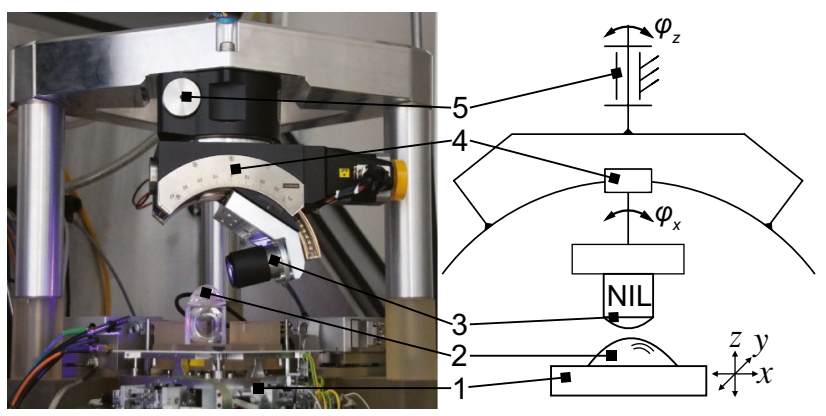

Fig. 8 NPMM [11] with rotating NIL tool; 1: linear stage of the $\operatorname{NPMM}(x, y, z), 2$ : plano-convex lens with substrate surface, 3: NIL tool, 4: goniometer stage $\left(\varphi_{x, y}\right)$ [19], 5: rotary stage $\left(\varphi_{z}\right)$ [18]
Table 1 Finite elements analysis (FEA) of the elastic deformation $(\varepsilon)$ and eigen-frequencies $\left(f_{\mathrm{e}}\right)$ of the reference hemisphere $(\mathrm{RH})$ by gravity in relation to material and wall thickness variation for representative materials with a minimal coefficient of thermal expansion

\begin{tabular}{lllllll}
\hline Properties & V1-1a & V1-1b & V1-2a & V2-1a & V2-1b & V2-2a \\
\hline Variant & Fused silica & & & Invar36 & & \\
Material & 400 & & & & & \\
Inner diameter $d_{\mathrm{i}-\mathrm{RH}}(\mathrm{mm})$ & 10 & 20 & $7.5-25$ & 10 & 20 & $7.5-25$ \\
Material thickness $\Delta r_{\mathrm{RH}}(\mathrm{mm})$ & 4.649 & 9.983 & 11.169 & 16.679 & 35.815 & 40.066 \\
Mass $m_{\mathrm{RH}}(\mathrm{kg})$ & 7.232 & 3.209 & 1.87 & 12.256 & 5.282 & 3.13 \\
Highest deformation $\varepsilon(\mathrm{RH})_{\max }(\mu \mathrm{m})$ & 0.209 & 0.175 & 0.161 & 0.423 & 0.307 & 0.291 \\
Lowest deformation $\varepsilon(\mathrm{RH})_{\min }(\mu \mathrm{m})$ & 151.84 & 179.9 & 295.8 & 113.3 & 142.14 & 226.55 \\
$f_{\mathrm{e} 1-\mathrm{RH}}(\mathrm{Hz})$ & 228 & 257.54 & 353.98 & 171.9 & 199.98 & 265.89 \\
$f_{\mathrm{e} 2-\mathrm{RH}}(\mathrm{Hz})$ & 260 & 347.54 & 430.66 & 189.96 & 271.33 & 329.7 \\
$f_{\mathrm{e} 3-\mathrm{RH}}(\mathrm{Hz})$ & & & & &
\end{tabular}




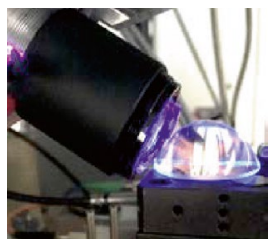

(a)

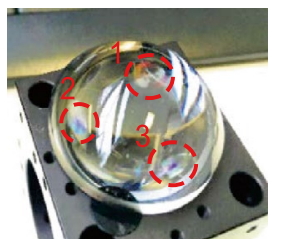

(b)

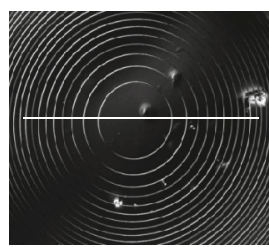

(c)

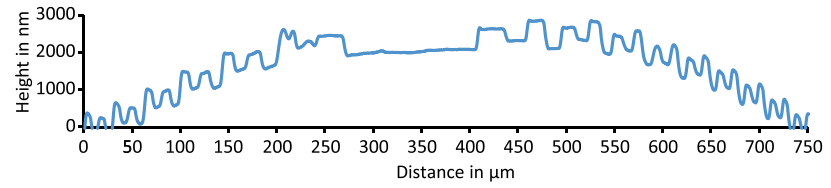

(d)

Fig. 9 NIL: a NIL curing phase at $45^{\circ}$ inclination to the horizontal, b multiple structured substrate on plano-convex lens, $\mathbf{c}$ image of structure $3\left(45^{\circ} \mathrm{NIL}\right.$ inclination to the horizontal) taken by means of a $3 \mathrm{D}$ laser scanning microscope [28], d profile of the structural cross section of the image $c$ with optical profiling system [29]

Combined movements of the axes are necessary to bring the tool in the printing position.

The object is uniformly coated with a substrate (AMONIL MMS4) [26] using the spray coating process [27]. The contact between the NIL tool and the substrate surface of the object is then made by a translational movement of the linear stage of the NPMM along the main tool axis. The contact of the stamp structures is followed by a resist filling time of 1 min for the stamp cavities, followed by a UV exposure time of $1 \mathrm{~min}$. After subsequent translational demolding along the main tool axis, the process can be repeated at other points depending on the viscosity of the substrate (Fig. 9).

Due to the strong curvature of the surface, the analysis of the generated structures is performed only for the center of the replicated overall structure (Fig. 9c, d). A comparison of the central region of this replicated structure with the original of the stamp shows good fidelity. Thus, in images taken with a 3D laser scanning microscope [28], the measured difference in structure heights between the stamp and the replicated structure for steps in the central region is in a range of 25 to $35 \mathrm{~nm}$.

The replication of structures over the entire object surface is not possible in a single printing process due to the strong curvature. Instead, the structuring of the entire object surface is carried out by a step and repeat printing process.

If continuous structuring is to be achieved, the partial structures must be linked to form an overall structure (stitching). For nanometer-precise stitching over the entire surface of the highly curved substrate, positioning with corresponding positioning accuracy of the stamp relative to the substrate is necessary.

Further investigations are therefore focused on the combination of the rotating NIL stamp with an in situ measurement of the displacements of the TCP, thus enabling the stitching of partial structures with nanometer precision.

\section{Conclusions}

A systematic parameter-based dynamic evaluation approach was developed for the creation and selection of adequate machine structures for multiaxial nanopositioning systems $(\mathrm{DOF}>3)$. Out of these investigations, a rotation of the tool is a favorable solution. Kinematics with a high degree of fulfilment consider a common instantaneous center of rotation in the tool center point (TCP). Compared to a fixed tool position, this leads to shifting deviations of the TCP due to deformations of the frame, positioning errors of the rotational axes, vibrations, and thermal influences.

However, a scientific novelty for nanofabrication processes is given by the possibility of addressing and processing three-dimensional bodies with a strong curvature normal to their surface. As exemplified with the application of a developed nanoimprint tool, the positioning properties are sufficient for nanofabrication processes without stitching.

With the overall design developed, different tools $\left(l_{T} \leq 60 \mathrm{~mm} ; m_{T} \leq 2 \mathrm{~kg}\right)$ can be tilted horizontally by up to $90^{\circ}$ and rotated $360^{\circ}$ around the vertical axis of the NPMM.

A commercially available NPMM of $25 \mathrm{~mm} \times 25 \mathrm{~mm} \times$ $5 \mathrm{~mm}(x, y, z)$ [11] translational movement of the object was chosen for experimental investigations. The developed setup allows the transfer to an NPMM of $200 \mathrm{~mm} \times 200 \mathrm{~mm} \times 25 \mathrm{~mm}(x, y, z)$ [1] translational movement for future investigations.

The strict separation of the force frame and the metrology frame, thermal shielding, and direct measuring systems for the deviation of the TCP can be implemented to compensate the effects that are dependent on the selected overall structure and positioning system. The in situ deviation measurement of the TCP around two axes simultaneously can be achieved considering a hemispherical concave mirror as reference in combination with compact laser interferometers.

A new type of overall structure developed, in which Fabry-Pérot interferometers are used for the combined measurement of positioning deviations, ensures that the rotations are included in the closed control loop, thus increasing the measurement and machining accuracy. The interferometers measure the positioning errors against a hemispherical concave mirror that is an inherent part of the metrological frame of the NPMM. Using a glass sphere as a retroreflector with a refractive index of two in the TCP ensures the in situ calibration of the TCP position. Long-term dimensional stability is a prerequisite for the hemisphere and the interferometer carrier as part of the metrological frame.

Future investigations are therefore focused on a modification of the developed setup by a reference hemisphere made of fused silica with reduced shape deviations and an increase of the orthogonality of the measuring axes as the major influencing variables for the total measurement uncertainty. 
Acknowledgements The authors gratefully acknowledge the support of the Deutsche Forschungsgemeinschaft (DFG) in the framework of Research Training Group "Tip- and laser-based 3D-nanofabrication in extended macroscopic working areas" (GRK 2182) at the Technische Universität Ilmenau, Germany

Funding Open Access funding enabled and organized by Projekt DEAL.

Conflict of interest Eberhard Manske is an editorial board member for "Nanomanfucturing and Metrology" and was not involved in the editorial review, or the decision to publish this article. All authors declare that there are no competing interests.

Open Access This article is licensed under a Creative Commons Attribution 4.0 International License, which permits use, sharing, adaptation, distribution and reproduction in any medium or format, as long as you give appropriate credit to the original author(s) and the source, provide a link to the Creative Commons licence, and indicate if changes were made. The images or other third party material in this article are included in the article's Creative Commons licence, unless indicated otherwise in a credit line to the material. If material is not included in the article's Creative Commons licence and your intended use is not permitted by statutory regulation or exceeds the permitted use, you will need to obtain permission directly from the copyright holder. To view a copy of this licence, visit http://creativecommons.org/licenses/by/4.0/.

\section{References}

1. Jäger G, Manske E, Hausotte T, Müller A, Balzer F (2016) Nanopositioning and nanomeasuring machine NPMM-200-a new powerful tool for large-range micro- and nanotechnology. Surf Topogr Metrol Prop 4(3):034004

2. IBS Precision Engineering (2015) ISARA400. Next generation ultraprecision coordinate measuring machine

3. SmarAct GmbH (2018) SMARPOD P-SLC-24. SMARPOD-a hexapod-like positioning system

4. Seggelen JK (2007) NanoCMM. A 3D coordinate measuring machine with low moving mass for measuring small products in array with nanometer uncertainty. Dissertation, Technische Universiteit Eindhoven

5. Schienbein R (2020) Grundlegende Untersuchungen zum konstruktiven Aufbau von Fünfachs-Nanopositionier- und Nanomessmaschinen. Dissertation, Technische Universität Ilmenau

6. Hausotte T (2011) Nanopositionier- und Nano-messmaschinen: Geräte für hochpräzise makro- bis nanoskalige Oberflächen- und Koordinatenmessungen. Habilitation. TU Ilmenau

7. Henselmans R (2009) Non-contact measurement machine for freeform optics. Dissertation, Technische Universiteit Eindhoven

8. AMETEK. Inc. Taylor Hobson (2019) LuphoScan 260-420 HD

9. Schuler A, Weckenmann A, Hausotte T (2014) Setup and evaluation of a sensor tilting system for dimensional micro- and nanometrology. J Meas Sci Technol 25(6):064010

10. Manske E, Jäger G (2012) Multi-sensor approach for multivalent applications in nanometrology. Int J Autom Smart Technol AUSMT 2:141-145

11. Jäger G, Manske E, Hausotte T, Büchner H-J (2009) The metrological basis and operation of nanopositioning and nanomeasuring machine NMM-1. TM Technisches Messen 76(5):524

12. Schienbein $R$, Theska $R$ (2017) A contribution to the development of multiaxial nanopositioning machines. In: Proceedings of the 17th international conference EUSPEN, Hannover, Germany, pp $113-114$

13. Schienbein R, Theska R, Weigert F (2017) A contribution to the implementation of ultraprecision rotations for multiaxial nanopositioning machines. In: Proceedings of the 59th Ilmenau scientific colloquium, Ilmenau, Germany

14. Verein Deutscher Ingenieure e.V. (1998) Konstruktionsmethodik Technisch-wirtschaftliches Konstruieren-Technisch-wirtschaftliche Bewertung

15. SmarAct GmbH, AEROTECH, INC. (2019) Physik Instrumente (PI) GmbH \& Co. KG, HUBER Diffraktionstechnik GmbH \& Co. KG, Newport Corporation (MKS Instruments), OWIS GmbH, MirconixUSA, Thorlabs Inc., Attocube systems AG

16. ARTAS-Engineering Software: SAM 7.1. www.artas.nl

17. Schienbein R, Fern F, Weigert F, Theska R, Füßl R (2018) The implementation of ultra precision rotations to multiaxial nanofabrication machines: challenges and solution concepts. In: Proceedings of the 18th international conference EUSPEN, Venice, Italy, pp 65-66

18. Physik Instrumente (PI) GmbH \& Co. KG (2019) L-611.9ASD precision rotation stage + SMC hydra motion controller

19. Physik Instrumente (PI) GmbH \& Co. KG (2019) WT-90 motorized precision goniometer + SMC hydra motion controller

20. Thorlabs GmbH (2019) CXYZ05/M-XYZ translation mount

21. Möller-Wedel-Optical (2019) Autokollimator Elcomat 3000

22. Fern F, Füßl R, Schienbein R, Theska R (2018) In: Proceedings of the 33rd American Society for precision engineering ASPE annual meeting, Las Vegas, pp 122-126

23. Attocube Systems-AG (2019) IDS3010 interferometric displacement sensor

24. Fern F (2020) Metrologie in fünfachsigen Nanomess- und Nanopositioniermaschinen. Dissertation, TU Ilmenau

25. Nichia Corp. (2019) NCSU275 UV SMD-LED with PCB $(10 \times 10 \mathrm{~mm}), 148 \mathrm{~mW}, 365 \mathrm{~nm}$

26. AMO GmbH (2019) AMONIL ${ }^{\circledR}$ : high performance UV nanoimprint resist

27. EV Group Europe \& Asia/Pacific GmbH (2019) EVG101 advanced resist processing system

28. OLYMPUS EUROPA SE \& CO. KG (2019) 3D-laserscanning Mikroskop (LSM, Olympus LEXT OLS4100)

29. Veeco Instruments Inc (2019) Wyko NT9100 optical profiling system

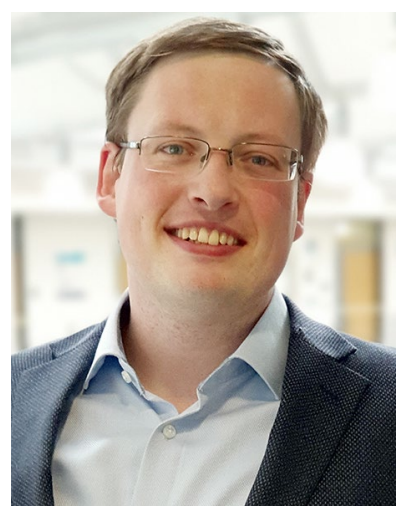

Ralf Schienbein studied Mechanical Engineering resulting in a M.Sc. in 2015 and the Ph.D. degree in 2020 in the field of Precision Engineering with a focus on multiaxial nanopositioning and nanomeasuring machines from the Technische Universität Ilmenau, Germany. Since 2015 he is a researcher at the Precision Engineering Group and since 2017 he works in the research training group "Tipand laser-based 3D-Nanofabrication in extended macroscopic working areas (NanoFab)" funded by the German Research Council. His research activities are particularly in the development of nanopositioning and nanomeasuring machines with a focus on high-precision machine design, systems engineering, interferometry and optical and tactile nano tools. 


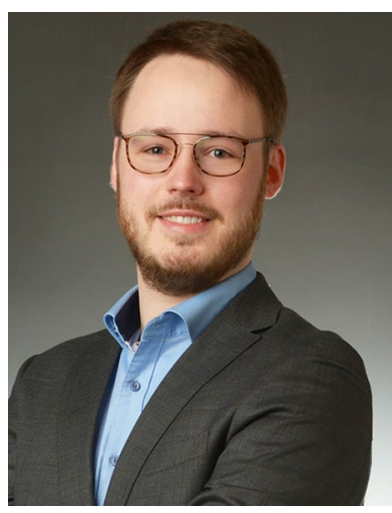

Florian Fern studied from 2012 to 2017 at the Technische Universität Ilmenau and received his master's degree in 2017. From 2017 to 2020 , he was a research associate at the Technische Universität Ilmenau at the institute Institute of Process Measurement and Sensor Technology and received his $\mathrm{Ph}$.D. with the thesis "Metrology in five axes nanomeasuring- and nanopositioning machines". Since 2020, he has been working as a research associate at JUMO $\mathrm{GmbH} \& \mathrm{Co}$. KG in the field of

sensors and control systems.

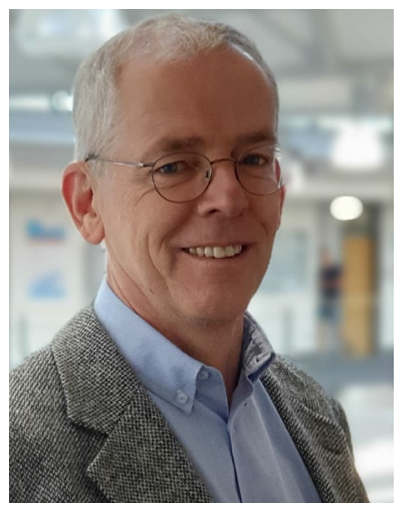

René Theska received the Diploma degree in 1983 and the Ph.D. degree in 1989 in Precision Engineering from the Technische Hochschule Ilmenau, Ilmenau, Germany. From 1989, he was with Leitz, Brown \& Sharpe Metrology Company, where he was in charge of the development of coordinate measuring machines and devices. From 2001, he is a full Professor at Technische Universität Ilmenau, Ilmenau, Germany. From 2008 to 2013 he was the deputy spokesman of the collaborative research centre "Nanopositioning and Nanomeasuring Machines (SFB 622)" and since 2017 he holds the same position in the research training group "Tip- and laser-based 3D-Nanofabrication in extended macroscopic working areas (NanoFab)" both funded by the German Research Council. His research interests are particularly in the development of nanopositioning and nanomeasuring machines as well as in force and toque measuring devices, with a focus on Precision Engineering.

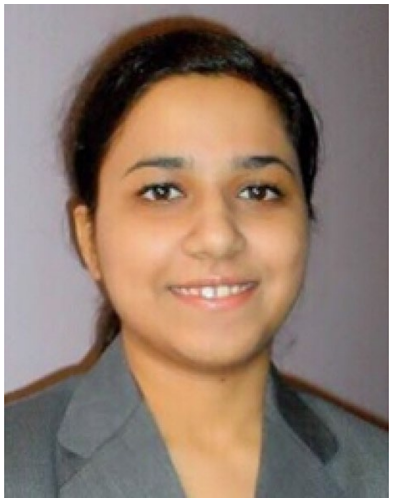

Shraddha Supreeti studied Smart Systems Integration at the Heriot-Watt University with stays at the Hogskolen in Buskerud, Vestfold and at the Budapest University of Technology and Economics and received her master's degree in 2015. From 2017 to 2020, she was a research associate at the Technische Universität Ilmenau at the Microsystems Technology Group and received her Ph.D. with the thesis "Soft NanoimprintLithography on CurvedSurfaces". Since 2020, she has been working as a research associate at the Institute of Microelectronics and Nanoelectronics as part of the Electronics Technology Group.

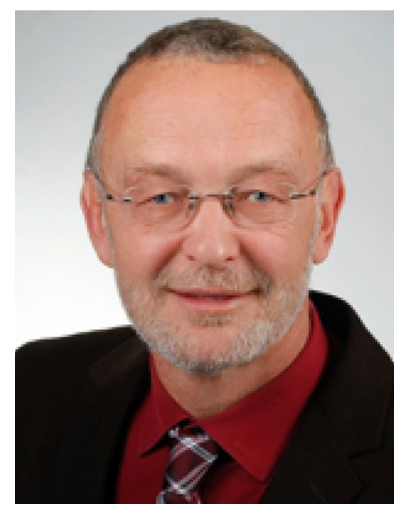

Roland FüßI received his diploma in technical cybernetics and automation technology from Ilmenau Technical University in 1981. He received his doctorate in 1986 and habilitated in 2008 in the field of process measurement technology. From 2008 to 2014 , he worked as a private lecturer at the Ilmenau University of Technology. In 2014, he was appointed as an associate professor at TU Ilmenau. From 2003 to 2013 he was a sub-project leader in the Collaborative Research Centre "Nanopositioning and Nanometrology Machines" and since 2017 he has been a project leader in the Research Training Group "Cutting-edge and Laser-based 3D Nanofabrication in Extended Macroscopic Workspaces". His research focuses on the field of metrology in nanopositioning-, nanomeasuringand nanofabrication technologies.

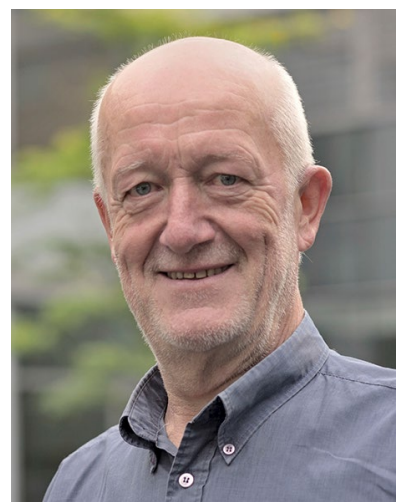

Eberhard Manske received the Diploma in Electrical Engineering from the Technische Universität Ilmenau in 1982. He obtained the Ph.D. degree in 1986, and in 2006 the postdoctoral lecturing qualification in the field of precision measurement technology. Since 2008 he holds a professorship "Production and Precision Measurement Technology" at the Technische Universität Ilmenau. From 2008 to 2013 he was spokesman of the collaborative research centre

"Nanopositioning and Nanomeasuring Machines (SFB 622)" and since 2017 he leads the research training group "Tip- and laser-based 3D-Nanofabrication in extended macroscopic working areas (NanoFab)" both funded by the German Research Council. His research activities are particularly in the development of nanopositioning and nanomeasuring machines with a focus on high-precision laser interferometry, laser stabilization, frequency comb technology, optical and tactile nano sensors and scanning probe techniques. 